\title{
TBC1D1 and TBC1D4 (AS160) RabGAP Domains are Characterized as Monomers in Solution by Analytical Ultracentrifugation
}

\author{
SangYoun Park ${ }^{\dagger}$ and Soon-Jong Kim* \\ Department of Chemistry, Mokpo National University, Muan 534-729, Korea. *E-mail: sjkim@mokpo.ac.kr \\ ${ }^{\dagger}$ School of Systems Biomedical Science Soongsil University, Seoul 156-743, Korea \\ Received April 19, 2011, Accepted May 5, 2011
}

Key Words : RabGAP domain, Crystal packing force distortion, Analytical ultracentrifugation

Upon food intake, the glucose level in circulating blood increases by trans-cellular transport of glucose from the intestinal lumen into the blood. However, the insulin secreted from the pancreatic $\beta$-cells directs the individual cells to absorb glucose for energy source to result in the maintenance of the normal blood glucose level. Elevated insulin in blood is sensed by the insulin receptor of the individual cells, and the propagation of the insulin signaling pathway drives the glucose uptake. The final cellular glucose uptake from the blood into the cells is performed by trafficking of vesicles containing the glucose transporter protein (e.g. GLUT4) from the intracellular pools to the cell surfaces. ${ }^{1}$ As in most of the vesicle budding, uncoating, docking and fusion processes, this translocation of GLUT4 vesicles are tightly regulated by Rab-GTPases (Rabs) and Rab.GTPase-activating proteins (RabGAPs). Rabs are GTPases with intrinsic activity, however RabGAPs enhance the low hydrolysis rate of Rab-bound GTP. ${ }^{2,3}$ The two major cell types that absorb glucose, which are the adipocytes and the skeletal myocytes, respectively encode TBC1D4 (also known as AS160) and TBC1D1 which are integral RabGAPs in the insulin-mediated GLUT4 vesicle trafficking event. ${ }^{4-6}$

TBC1D4 and TBC1D1 are multi-domain RabGAP proteins which consist of two putative N-termini phosphotyrosine binding (PTB) domains and a C-terminal catalytic RabGAP domain that are linked by regions containing several Akt(PKB)-mediating serine and threonine phosphorylation sites. In the basal state, TBC1D4 (or TBC1D1)-catalyzed hydrolysis of Rab-bound GTP (active) to GDP (inactive) leaves GLUT4 vesicles sequestered in an intracellular compartment. However, insulin signal results in the activation of Akt (PKB) and the consequent phosphorylation of TBC1D4 (or TBC1D1). The Akt phosphorylation of TBC1D4 (or TBC1D1) deactivates the Rab.GTP hydrolysis activity by an unknown mechanism, and concomitantly results in the increase of Rab-GTP concentrations. The active Rab·GTP in turn promotes GLUT4 vesicle translocation to the cell membrane and finally stimulates the glucose uptake from blood into the cells.

X-ray crystal structures of the human TBC1D1 RabGAP domain and TBC1D4 RabGAP domain (PDB code: 3QYE $\& 3 \mathrm{QYB}$, respectively) have been recently reported. ${ }^{7}$ Since, TBC1D1 and TBC1D4 share 76\% sequence identity over their RabGAP domains, the secondary structure elements mostly overlapped in the two structures which were predominately $\alpha$-helical with no $\beta$-sheet elements. The $17 \alpha$ helices in TBC1D1 RabGAP domain were sequentially named from $\alpha 1$ to $\alpha 16$ ending with $\alpha 16$ '. However, the oligomeric association states of the two TBC1D1 and TBC1D4 RabGAP domains in the crystal asymmetric unit were different resulting from the presence (or absence) of a single helix $\alpha 16$ '. TBC1D1 RabGAP domain containing the C-terminal $\alpha 16^{\prime}$ forms an asymmetric dimer with $\alpha 16^{\prime}$ of one molecule interacting with $\alpha 16$ of the other molecule (Figure 1). However in TBC1D4 RabGAP domain, $\alpha 16^{\prime}$ in the protein construct had to be deleted for crystallization and enhanced X-ray diffraction (Figure 2), and this results in TBC1D4 RabGAP domain being a monomer in the crystal (Figure 1). Certain is the fact that $\alpha 16$ ' mediates the resulting differences in the oligomeric states, the biological relevance of this asymmetric dimer cannot be concluded only from the association modes within the crystal. Since only two $\alpha$ helices $\left(\alpha 16^{\prime}\right.$ and $\left.\alpha 16\right)$ of TBC1D1 mediate the dimerization, it was suspected that the dimer is a result of crystal packing artifacts, and is non-relevant outside the context of the crystal. However in this study, we have compared the oligomeric association states of TBC1D1 and TBC1D4 RabGAP domains (Figure 2) using analytical ultracentrifugation (AUC) techniques to address the state of interaction in solution.

Sedimentation equilibrium experiments were performed in a Beckman-Coulter ProteomeLab XL-A analytical ultracentrifuge using $12 \mathrm{~mm}$ carbon-filled epoxy double-sector centerpieces and quartz windows at optical density of 0.25 at $280 \mathrm{~nm}(6.67 \mu \mathrm{M}$ and $6.69 \mu \mathrm{M}$ for TBC1D1 and TBC1D4 RabGAP domains, respectively). The measurements were performed at two different rotor speeds of 13,000 and 18,000 RPMs at $20^{\circ} \mathrm{C}$. The proteins were in $50 \mathrm{mM}$ Tris- $\mathrm{HCl}, \mathrm{pH}$ $7.5,0.15 \mathrm{M} \mathrm{NaCl}$, and $1.5 \mathrm{mM}$ TCEP. The sedimentation equilibrium data were analyzed using MLAB software (Civilized Software, Inc., Silver Spring, MD) using the following equation ${ }^{8}$ :

$$
\mathrm{C}(\mathrm{r})=c_{b} * \exp \left(\mathrm{A}_{\mathrm{p}} * \mathrm{M}_{\mathrm{p}} *\left(\mathrm{r}^{2}-\mathrm{r}_{\mathrm{b}}^{2}\right)+\varepsilon\right.
$$

where $\mathrm{C}(\mathrm{r})$ represents the total absorbance at radius $\mathrm{r}$ and $c_{b}$ is the absorbance of at $r_{b}$, the radius at the cell bottom, which 
(a)

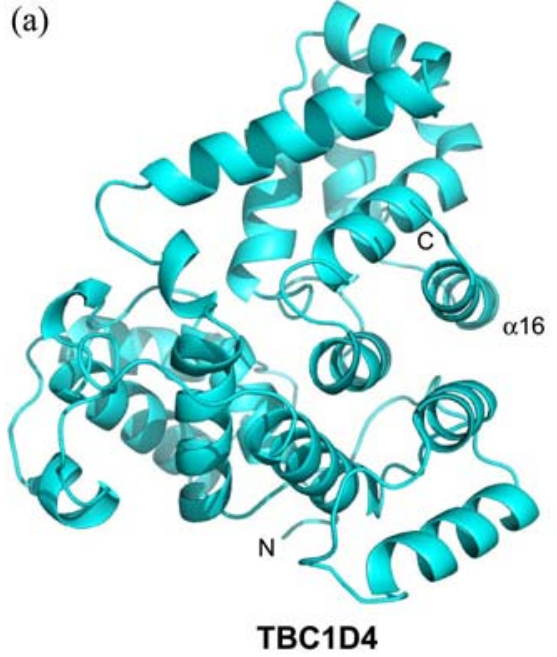

(b)

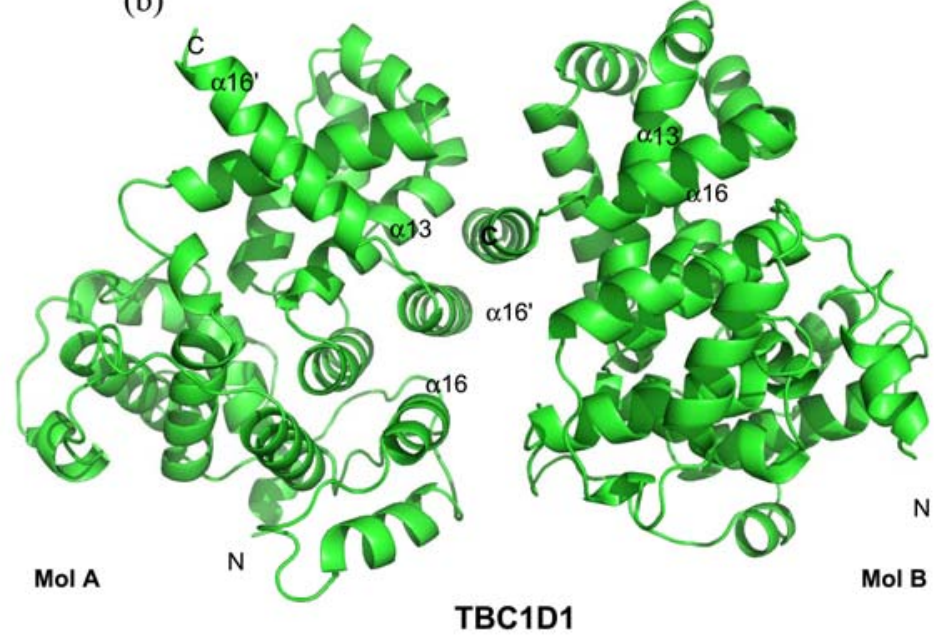

(c)

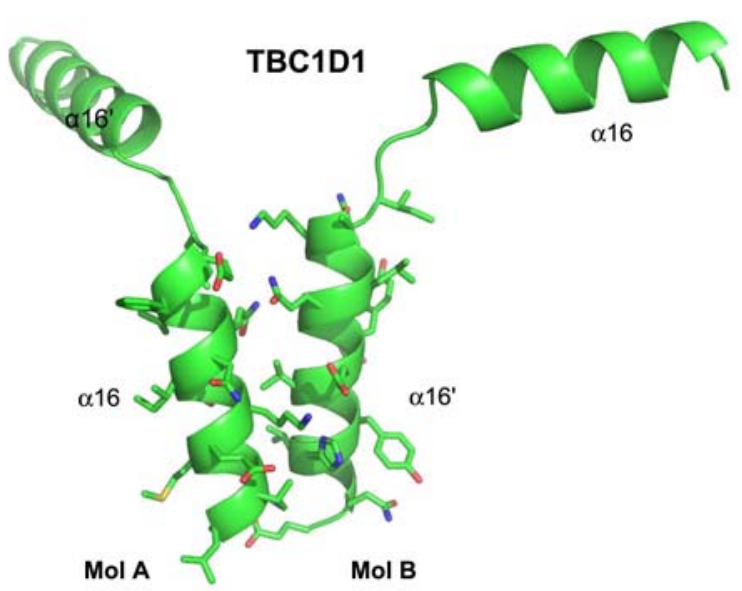

Figure 1. Overall crystal structures of TBC1D1 and TBC1D4 RabGAP domain in the crystal asymmetric unit. (A) Only one molecule of TBC1D4 RabGAP domain is observed in the crystal asymmetric unit of TBC1D4 RabGAP domain. For determination of the TBC1D4 RabGAP domain structure, $\alpha 16$ ' which mediates dimer formation in TBC1D1 had to be deleted from the construct. N-terminal and Cterminal are indicated by "N" and " $\mathrm{C}$ " respectively. (B) Analysis of the two molecules of TBC1D1 RabGAP domain in the crystal asymmetric unit indicates an asymmetric dimer. The dimer is mediated by the interaction of $\alpha 16$ (Mol A) and $\alpha 16$ ' (Mol B). N-terminals and $\mathrm{C}$-terminals of the two molecules are indicated by " $\mathrm{N}$ " and " $\mathrm{C}$ " respectively. (C) Detailed side chains of $\alpha 16$ and $\alpha 16$ ' interface between the two TBC1D1 RabGAP domain molecules are shown with other regions not included for clarity.

is used as a reference point. $A_{p}$ is $(1-\bar{v} \rho) \omega^{2} / 2 R T$, where $\bar{v}$ is the partial specific volume of the respective proteins, $\rho$ is the density of the solvent at a given temperature, $\omega$ is the angular velocity of the rotor (in radians $\mathrm{s}^{-1}$ ), $\mathrm{R}$ is the universal gas constant, $\mathrm{T}$ is the absolute temperature (293.15 $\mathrm{K}), \mathrm{M}_{\mathrm{p}}$ is the molecular mass of the protein monomers (40,298 and 40,503 daltons for TBC1D1 and TBC1D4 RabGAP domains including N-terminal histidine tags, respectively), and $\varepsilon$ is the baseline correction. Using the Eq. (1), AUC data obtained were fitted to models assuming various homogeneous forms of monomer (1x), dimer (2x) and trimer (3x). Results of equilibrium sedimentation analyses for the oligomeric state of TBC1D1 RabGAP domain are shown in Figure 3. For the TBC1D1 RabGAP domain, the root-mean-square error (RMS) for the monomer (1x) fit was $8.54 \times 10^{-3}$ demonstrating the goodness of the model. In contrast, trials to fit the data to a dimer $(2 \mathrm{x})$ and a trimer $(3 \mathrm{x})$ models showed systematic deviations with RMS values of $4.47 \times 10^{-2}$ and $7.86 \times 10^{-2}$, respectively. The overall data demonstrate that TBC1D1 RabGAP domain exists as a homogeneous monomer in solution. Additional equilibrium sedimentation analyses for the homologous TBC1D4 RabGAP domain are also shown in Figure 4. The RMS values for $1 \mathrm{x}, 2 \mathrm{x}$ and $3 \mathrm{x}$ fits were $9.30 \times 10^{-3}, 3.95 \times 10^{-2}$ and $7.03 \times 10^{-2}$, respectively suggesting that TBC1D4 RabGAP domain also exists as a monomer in solution. Other interactive or mixture models were also tested using the data collected at two different speeds but there was no indication of other possibilities.

In the crystal lattice, two TBC1D1 RabGAP domains form an asymmetric dimer which is mediated by only a single $\alpha$ helix contribution from each molecule (Figure 1). When the dimeric TBC1D1 structure (PDB code: 3QYE) was analyzed with solvent-accessible surface area calculation pro- 


\section{PTB PTB RabGAP} $\begin{array}{lc}\text { TBC1D4 RabGAP } & \sqrt[865]{\text { RabGAP }}{ }^{1191} \\ \text { TBC1D4 RabGAP* } & \sqrt[76]{\text { RabGAP }}{ }^{1072}\end{array}$

\begin{tabular}{|c|c|c|c|c|}
\hline $\begin{array}{c}\text { RabGAP } \\
\text { proteins }\end{array}$ & $\begin{array}{c}\text { Sequence } \\
\text { (Secondary structure) }\end{array}$ & $\begin{array}{c}\text { Crystal } \\
\text { diffraction }\end{array}$ & $\begin{array}{c}\text { No. in } \\
\text { crystal AU }\end{array}$ & $\begin{array}{c}\text { No. from } \\
\text { AUC }\end{array}$ \\
\hline TBC1D4 $^{\circ}$ & $865-1191\left(\alpha 1-\alpha 16-\alpha 6^{\prime}\right)$ & No good & NA & 1 \\
\hline TBC1D4 & $873-1172(\alpha 1-\alpha 16)$ & Good & 1 & NA \\
\hline TBC1D1;,- & $746-1072\left(\alpha 1-\alpha 16-\alpha 16^{\prime}\right)$ & Good & 2 & 1 \\
\hline
\end{tabular}

'Protein constructs used for the analysis.

"Sequence identity is 76\% between TBC1D1 and TBC1D4 RabGAP domains

Figure 2. Comparisons of the two TBC1D1 and TBC1D4 RabGAP domains used for the AUC analysis. TBC1D1 and TBC1D4 (AS160) are a multi-domain proteins with two N-termini PTB domains and a C-terminal catalytic RabGAP domain. We have used two constructs of TBC1D1 and TBC1D4 RabGAP domains for the AUC experiments, which are tabulated with crystallographic and solution AUC results. The two constructs used for AUC both contain the $\alpha 16$ ' which mediates dimer formation in the crystal structure of TBC1D1 RabGAP domain. For the determination the TBC1D4 RabGAP domain structure, $\alpha 16$ ' was deleted from the construct which was necessary for enhanced Xray diffraction.

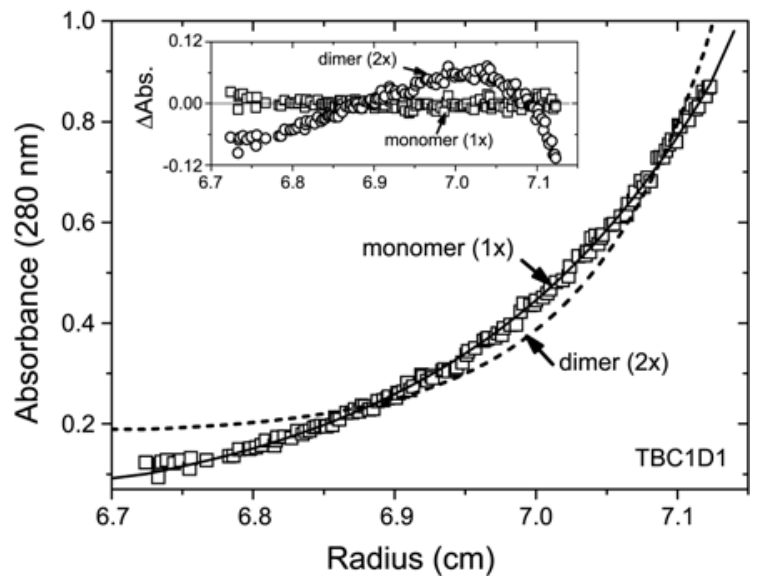

Figure 3. Sedimentation equilibrium distribution of TBC1D1 RabGAP domain at $13,000 \mathrm{rpm}\left(20^{\circ} \mathrm{C}\right)$. The protein concentration of $6.67 \mu \mathrm{M}(0.28 \mathrm{mg} / \mathrm{mL})$ was used. The squares represent the experimental data at $280 \mathrm{~nm}$ and the solid and dotted lines are fitted lines for the ideal monomer $(1 \mathrm{x})$ and dimer $(2 \mathrm{x})$ models, respectively. The calculated molecular mass for TBC1D1 RabGAP domain monomer (1x) including the N-terminal His-tag is 40,298 daltons. (Inset) Distributions of the residuals for the monomer (1x, square) and the dimer $(2 \mathrm{x}$, circle). The random distributions of residuals for the monomer $(1 \mathrm{x})$ model indicate that TBC1D1 RabGAP domain exists as a homogeneous monomer in solution.

grams such as AreaIMol $^{9}$ (or GetArea ${ }^{10}$ ), the area that is buried upon dimer formation was $890 \AA^{2}$ (or $790 \AA^{2}$ ) per monomer molecule by the two respective methods. From a recent study using a non-redundant structure database, it was reported that no known physiological dimer has a contact surface area of less than $500 \AA^{2}$ and the optimum cut-off for discriminating monomers and dimers was $856 \AA^{2}{ }^{11}$ Since the calculated solvent-accessible surfaces for the TBC1D1 RabGAP domain dimer are close to the optimal cut-off

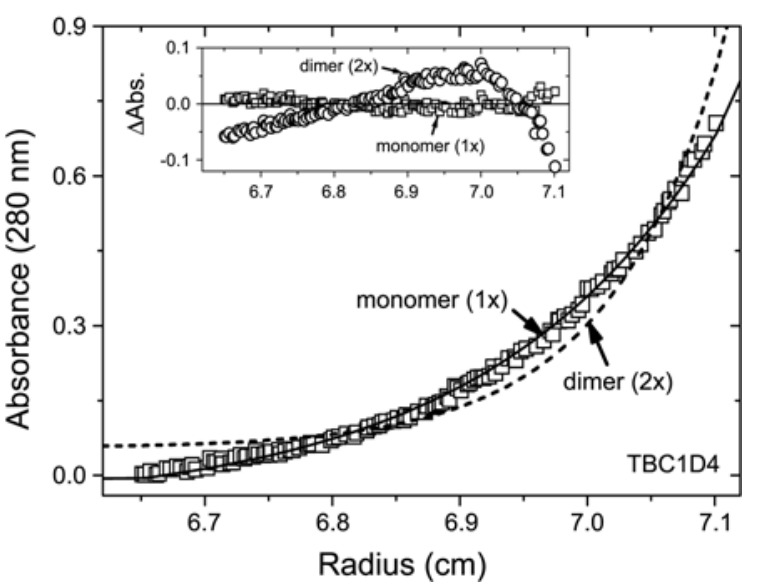

Figure 4. Sedimentation equilibrium distribution of TBC1D4 RabGAP domain at $13,000 \mathrm{rpm}\left(20^{\circ} \mathrm{C}\right)$. The protein concentration of $6.69 \mu \mathrm{M}(0.27 \mathrm{mg} / \mathrm{mL})$ was used. The squares represent the experimental data at $280 \mathrm{~nm}$ and the solid and dotted lines are fitted lines for the ideal monomer $(1 \mathrm{x})$ and dimer $(2 \mathrm{x})$ models, respectively. The calculated molecular mass for TBC1D4 RabGAP domain monomer $(1 \mathrm{x})$ including the N-terminal His-tag is 40,503 daltons. (Inset) Distributions of the residuals for the monomer (1x, square) and the dimer $(2 \mathrm{x}$, circle). The random distributions of residuals for the monomer $(1 \mathrm{x})$ model indicate that TBC1D4 RabGAP domain also exists as a homogeneous monomer in solution.

value, it is important to determine whether the dimeric arrangement is relevant in solution or is just a crystal packing artifact. However, the results of sedimentation equilibrium experiments indicated that TBC1D1 and TBC1D4 RabGAP domains are essentially monomers as shown in Figure 3 and Figure 4, respectively. Velocity sedimentation experiments carried out using much higher protein concentrations also showed a single peak corresponding to the molecular weights of the monomeric RabGAP domains (data not shown here). Based on all of our AUC analyses, it is clear that the dimerization of TBC1D1 RabGAP domain is coming from crystal packing artifacts and is not relevant outside the crystal lattice. The crystallization induced artifacts in protein crystals are rather frequent and it is advisable to confirm the oligomeric states in solution by proper analytical methods such as the AUC techniques. ${ }^{12-14}$

In summary, we have shown that despite human TBC1D1 RabGAP domain existing as an asymmetric dimer in a crystal lattice, two RabGAP domains of human TBC1D1 and TBC1D4 investigated here behave as homogeneous monomers in solution (Figures $3 \& 4$ ). Hence, caution should be exercised whenever the biological homo-oligomerization states of proteins are concluded from interaction modes within a crystal structure. Since oligomer interactions observed in the crystal can originate from the packing artifacts, it would be worthwhile to confirm (or disconfirm) the crystal association states in solution by using analytical methods such as the ultracentrifugation. It is also noticeable that the dimer artifact in TBC1D1 RabGAP domain in the crystal structure was cause by a single extra helix ( $\left.\alpha 16^{\prime}\right)$. Needless to say, homo-oligomerization into multimeric proteins is a key feature in many biological systems for added 
complexity. The higher order of quaternary structure often provides synergistic regulation and enhances the functional properties especially in the multi-enzyme complex. Since TBC1D4 and TBC1D1 are multi-domain proteins of $\sim 1300$ residues, there is ample possibility that it may form homooligomers via domains or regions. This possibility is under investigation using AUC methods. However in this study we confirm that the catalytic RabGAP domains by themselves do not mediate the oligomerization.

Acknowledgments. This work was supported by the startup funds from Soongsil University, Seoul, Korea to Park SY and by a research fund (2007) of Mokpo National University, Muan, Korea to Kim S.-J.

\section{References}

1. Watson, R. T.; Pessin, J. E. Trends Biochem. Sci. 2006, 31, $215-$ 222.

2. Zerial, M.; McBride, H. Nat. Rev. Mol. Cell Biol. 2001, 2, 107119.

3. Stenmark, H. Nat. Rev. Mol. Cell Biol. 2009, 8, 513-525.

4. Kane, S.; Sano, H.; Liu, S. C. H.; Asara, J. M.; Lane, W. S.; Garner, C. W.; Lienhard, G. E. J. Biol. Chem. 2002, 277, 22115-
22118.

5. Sano, H.; Kane, S.; Sano, E.; Mînea, C. P.; Asara, J. M.; Lane, W. S.; Garner, C.W.; Lienhard, G. E. J. Biol. Chem. 2003, 278, 14599 14602

6. Sano, H.; Roach, W. G.; Peck, G. R.; Fukuda, M.; Lienhard, G. E. Biochem. J. 2008, 411, 89-95.

7. Park, S. Y.; Jin, W.; Woo, J. R.; Shoelson, S. E. J. Biol. Chem. 2011.

8. Jung, W. S.; Hong, C. K.; Lee, S.; Kim, C. S.; Kim, S.-J.; Kim, S. I.; Rhee, S. J. Biol. Chem. 2007, 282(11), 8414-8423.

9. Collaborative Computational Project, Number 4. The CCP4 Suite: Programs for Protein Crystallography; Acta Cryst. 1994, D50, 760-763.

10. Fraczkiewicz, R.; Braun, W. J. Comp. Chem. 1998, 19, 319-333.

11. Ponstingl, H.; Henrick, K.; Thornton, J. M. Proteins 2000, 41, 47 57.

12. Wang, W. K.; Bycroft, M.; Foster, N. W.; Buckle, A. M.; Fersht, A. R.; Chen, Y. W. Acta Cryst. 2001, D57, 545-551.

13. Wang, Y.; Coulombe, R.; Cameron, D. R.; Thauvette, L.; Massariol, M. J.; Amon, L. M.; Fink, D.; Titolo, S.; Welchner, E.; Yoakim, C.; Archambault, J.; White, P. W. J. Biol. Chem. 2004, 279, 69766985.

14. Lee, H. H.; Kim, Y. S.; Kim, K. H.; Heo, I.; Kim, S. K.; Kim, O.; Kim, H. K.; Yoon, J. Y.; Kim, H. S.; Kim, D. J.; Lee, S. J.; Yoon, H. J.; Kim, S. J.; Lee, B. J.; Song, H. K.; Kim, V. N.; Park, C. M.; Suh, S. W. Mol. Cell 2007, 27, 938-950. 